\title{
The role of MRI in pancreatic cancer
}

Published online: 16 October 2001

(C) Springer-Verlag 2001

The treatment of pancreatic cancer remains problematic. Imaging procedures for diagnosis and staging are currently directed at identifying those patients who may be suitable for curative surgery. In this issue, Fischer et al. [1] argue a case for using MRI as a single staging examination in potential surgical candidates. The content and the implications of their paper raise several questions for further discussion.

Firstly, is the particular MRI technique which they describe really the most appropriate for assessment of pancreatic cancer? Secondly, can we be confident that MRI is superior to CT? Thirdly, like other radiology authors, they have focused on the determination of resectability, but what actually is meant by "resectable"? Finally, the contribution of imaging in this disease has to be judged against the outcome of attempted cure, so what are the factors which now hinder successful treatment, and how might imaging be able to help?

P.J.A. Robinson (

Department of Clinical Radiology, St. James's University Hospital, Beckett Street, Leeds LS9 7TF, UK Tel.: +44-113-2064724

Fax: +44-113-2064691

\section{MRI techniques for pancreatic cancer}

Initial breath-hold MRCP acquisitions show the site of ductal obstruction or stenosis. Further sequences can then be targeted to the location of the tumour and the adjacent vessels and other structures. Using a dedicated MRA technique after gadolinium, as Fischer et al. have done, gives an elegant demonstration of vascular anatomy, as their images show; however, the conspicuity of pancreatic carcinomas is greatest during the capillary phase of enhancement, so if T1-weighted images through the lesion are obtained only after the venous phase of MRA, they will be too late for the optimum visualisation of small tumours. The demonstration of liver metastases also requires sequential acquisitions during the arterial-dominant and portal venous phases of contrast enhancement, which cannot be achieved in the same examination as a detailed MRA acquisition through the coeliac/mesenteric area. A compromise which may be preferable is to use a 3D-volume technique (e.g. the VIBE sequence) for consecutive acquisitions in the late arterial/capillary phase, and the portal venous phase. A single 3D breath-hold acquisition will cover the liver and pancreas with 2- to 3-mm partitions in virtually all patients, particularly if a right anterior/coronal view is chosen to maximise the cranio-caudal coverage, while retaining the whole of the liver within the field of view. Using this approach, a simultaneous demonstration of vessels, normal parenchyma, and hypointense tumour can be achieved. Frequencyselective fat saturation can be added to improve tumour and vascular contrast, without imposing a significant sacrifice in terms of coverage or slice thickness. This sequence is probably also the best currently available for the difficult task of detecting peritoneal disease.

\section{Is MRI superior to CT?}

Early MRI studies using spin-echo techniques produced results which were inferior to dynamic contrastenhanced CT for detecting and staging pancreatic carcinoma. The introduction of dynamic gadoliniumenhanced gradient-echo sequences led to considerable improvement in the accuracy of MRI. More recently, a direct comparison of multiphase enhanced MRI with dual-phase helical CT [2] found MRI to be significantly better for assessment of vascular invasion, although the techniques were equally accurate in detecting the tumours.

The value of these comparative studies is relatively short-lived because of the rapid progress in imaging technology. The differences in 
accuracy between CT and MRI for pancreatic cancer are small enough to be overtaken by new technologies. This point is illustrated by a recent study [3] which found CT to be superior to MRI in demonstrating vascular invasion, but while these authors used 3-mm collimation with 2-mm reconstructions for $\mathrm{CT}$, they used 8-mm slices for MRI acquisitions. Most recently, multi-detector, multi-slice CT has given us the opportunity to carry out dual- or triplephase acquisitions through the pancreas with effective slice thickness in the range of 1-2 $\mathrm{mm}$, while the development of 3D volume acquisitions for MRI offers a parallel improvement in spatial and temporal resolution. Some further improvement in the accuracy of pre-operative detection and staging may be expected, but in pancreatic cancer all imaging methods face more problematic limitations.

\section{What is meant by "resectable"?}

Like other radiological authors, Fischer et al. [1] have concentrated their imaging technique on demonstrating whether or not the main vascular structures (portal vein, superior mesenteric vein and superior mesenteric artery) are infiltrated directly by the adjacent tumour. Good visualisation of the vessels can be achieved using either MRI or helical CT, but the interpretation of the images in terms of "resectability" is not always clear. In the majority of cases, where the vessels are either clearly normal, stenosed, occluded or completely encased by tumour, the interpretation is straightforward. In a minority of cases, the radiologist will be faced with the same difficulty as the surgeon at the time of laparotomy: What degree of vascular involvement is enough to rule out an attempt at curative resection? This difficulty may account for the results of Fischer et al. [1] who found that 5 of 12 patients whom they interpreted as unresectable on MRI underwent successful surgical treatment, and vascular invasion was not confirmed. Clearly, "resectability" in these cases is a decision for the surgeon, not the radiologist.

What we need is for radiologists and surgeons to agree on detailed and specific criteria for non-resectability. In terms of venous invasion, which appears to be the most problematic area for assessment, Spencer et al. [4] defined unresectable involvement as tumour encompassing more than $180^{\circ}$ of circumference of the superior mesenteric or portal vein over an axial length of $2 \mathrm{~cm}$ or more, or local stenosis/occlusion. Using this scheme, pre-operative MRI was confirmed by surgical findings in almost all cases. Similarly, grading schemes for degrees of vascular involvement were used by $\mathrm{Lu}$ et al. [5] and O'Malley et al. [6] who were able to define imaging criteria which could predict surgical findings with an accuracy of $90 \%$ or more. Using such detailed criteria, we should be able to predict the degree of vascular involvement found at surgery by using pre-operative MRI, and leave the decision about resectability to the surgeon.

In addition to vascular involvement, the surgical criteria for non-resectability include direct local invasion into retroperitoneal structures, and metastases to the liver, peritoneum or distant lymph nodes. In most surgical specimens with lymph node involvement, the majority of metastatic nodes are smaller than $1 \mathrm{~cm}$. Pre-operative recognition of these lymph node metastases by MR or CT is currently unreliable. Peritoneal disease is apparent on imaging only at an advanced stage, so in those patients with small serosal deposits the demonstration of non-resectability is made only at the time of surgery. The presence of liver metastases immediately rules out the possibility of curative surgery, so our imaging assessment must include examination of the liver. Again, the sensitivity of imaging techniques for detecting liver lesions smaller than $1 \mathrm{~cm}$, particu- larly on the liver surface, remains relatively poor. In colorectal cancer patients, Ward et al. [7] found MRI detected approximately $60 \%$ of such small lesions, whereas only approximately $45 \%$ were found on helical CT. With all these limitations, imaging cannot exclude early metastatic disease, so the decision to attempt curative resection continues to be made at surgery; however, even when resection is technically feasible, the results of surgery are still disappointing.

\section{The results of "curative" resection}

Several recent surgical series have focused on the outcome of patients with pancreatic cancer in whom curative resection has been attempted, and on the factors limiting successful treatment. Since the decision to resect is made by the individual surgeon at the time of operation, there is bound to be some variation in the criteria used to select patients for attempted curative resection. With this reservation, it is clear from recent surgical reports that the mortality of the operation itself should be approximately $5 \%$ or less, but the eventual outcome remains poor. Five-year survival rates for patients in whom "curative" resection has been performed vary between 10 and $20 \%$, with median survival of 12-18 months. Poorly differentiated histology (found in approximately one-third of cases), positive resection margins (present in up to $30 \%$ of cases) and tumour size greater than $2-3 \mathrm{~cm}$ are all associated with worse prognosis. In large surgical series, over $70 \%$ of patients in whom curative resection was carried out were found to have malignant invasion of local lymph nodes [8,9]. Even in those patients with negative nodes, invasion of the lymphatic vessels is commonly seen. Intrapancreatic perineural invasion, also found histologically in a substantial proportion of cases, is a further ad- 
verse prognostic finding. The patients in whom the best outlook is achieved are those with tumours smaller than $2 \mathrm{~cm}$, without nodal, serosal or retroperitoneal invasion; however, this subgroup accounts for only a tiny proportion of all pancreatic cancers (approximately $2 \%$ in a recent series of 670 cases [10]). The only one of these prognostic features which can be determined reasonably accurately by non-invasive imaging techniques is tumour size. The other factors which prevent successful surgical treatment are all related to histological grading or local invasion at microscopic level. It is difficult to see how we can improve this dismal situation with current imaging techniques, and for now we can only aim to define the gross anatomy of the lesion, assess the major vessels and detect distant metastases of measurable size.

\section{References}

1. Fischer U, Vosshenrich R et al. (2001) Preoperative local MRI-staging of patients with a suspected pancreatic mass. Eur Radiol DOI 10.1007/s00330-001-1149-5

2. Sheridan MB, Ward J et al. (1999) Dynamic contrast-enhanced MR imaging and dual-phase helical CT in the preoperative assessment of suspected pancreatic cancer: a comparative study with ROC analysis. Am J Roentgenol 173:583-590

3. Nishiharu T, Yamashita Y et al. (1999) Local extension of pancreatic carcinoma: assessment with thin-section helical CT versus with breath-hold fast MRI - ROC analysis. Radiology 212:445-452
4. Spencer JA, Ward J et al. (1998) Assessment of resectability of pancreatic cancer with dynamic contrast-enhanced MRI: technique, surgical correlation and patient outcome. Eur Radiol 8:23-29

5. Lu DSK, Reber HA et al. (1997) Local staging of pancreatic cancer: criteria for unresectability of major vessels as revealed by pancreatic-phase, thin-section helical CT. Am J Radiol 168:1439-1443

6. O'Malley ME, Boland GW et al. (1999) Adenocarcinoma of the head of the pancreas: determination of surgical unresectability with thin-section pancreatic-phase helical CT. Am J Radiol 173:1513-1518

7. Ward J, Naik KS et al. (1999) Hepatic lesion detection: comparison of MRI after the administration of superparamagnetic iron oxide with dual-phase CT by using alternative free-response ROC analysis. Radiology 210:459-466
8. Sohn TA, Yeo CJ et al. (2000) Resected adenocarcinoma of the pancreas 616 patients: results, outcomes and prognostic indicators. J Gastrointest Surg 4:567-579

9. Meyer W, Jurowich C et al. (2000) Pathomorphological and histological prognostic factors in curatively resected ductal adenocarcinoma of the pancreas. Surg Today 30:582-587

10. Birk D, Fortnagel G et al. (1998) Small carcinoma of the pancreas. Factors of prognostic relevance. J Hepatobiliary Pancreat Surg 5:450-454 